Article

\title{
Effect of Twisting and Stretching on Magneto Resistance and Spin Filtration in CNTs
}

\author{
Anil Kumar Singh * (D) and Sudhanshu Choudhary \\ National Institute of Technology Kurukshetra, Kurukshetra 136119, India; sudhanshu@nitkkr.ac.in or \\ hellosudhanshubit@gmail.com \\ * Correspondence: anil.nit.kr@gmail.com; Tel.: +91-9138-106-238
}

Received: 10 April 2017; Accepted: 20 August 2017; Published: 24 August 2017

\begin{abstract}
Spin-dependent quantum transport properties in twisted carbon nanotube and stretched carbon nanotube are calculated using density functional theory (DFT) and non-equilibrium green's function (NEGF) formulation. Twisting and stretching have no effect on spin transport in CNTs at low bias voltages. However, at high bias voltages the effects are significant. Stretching restricts any spin-up current in antiparallel configuration (APC), which results in higher magneto resistance (MR). Twisting allows spin-up current almost equivalent to the pristine CNT case, resulting in lower MR. High spin filtration is observed in PC and APC for pristine, stretched and twisted structures at all applied voltages. In APC, at low voltages spin filtration in stretched CNT is higher than in pristine and twisted ones, with pristine giving a higher spin filtration than twisted CNT.
\end{abstract}

Keywords: twisted carbon nanotube (twisted CNT); stretched carbon nanotube (stretched CNT); magneto resistance (MR); spin efficiency

\section{Introduction}

Spintronics involves investigations associated with the electron's spin, magnetic moment, and charge. Magnetic junction has usefulness in magnetic device applications, for example, magneto-resistive random access memory (MRAM), read heads of hard disk drives, programmable logic, and so on [1,2]. A two probe magnetic junction is a three-layer device in which nonmagnetic material is sandwiched between two electrodes. Electrodes are basically made up of ferromagnetic materials. The ferromagnetic material is chosen because of its unique electronic properties; that is, that spin-up and spin-down electrons pass from one electrode to other electrode through the magnetic junction with different Fermi energies. High tunnel magnetoresistance and high spin filtration are required for a CNT-based spintronic device to deliver better performance and ensure its use in spin valves and other spintronic-based devices. The above requirements can be satisfied by the use of half-metallic ferromagnets with $100 \%$ polarization as electrodes [3]. Though dozens of systems have been predicted to be half-metallic, $\mathrm{CrO}_{2}$ is essentially the only material that has been experimentally shown to definitively be a half-metal [4]. Magnetic tunnel junctions were experimentally fabricated using thin films of the half-metallic ferromagnet $\mathrm{CrO}_{2}$, employing $\mathrm{SnO}_{2}$ tunnel barriers in [5].

Carbon nanotube (CNT) was discovered in the 1990s [6]. CNT has high electrical conductivity and thermal conductivity. It can be metallic or a semiconductor [7-10]. Carbon nanotubes (CNTs) can be used in spintronic devices due to their nanoscale size and very large spin flip scattering lengths [2]. Spin can be transported in CNTs over large lengths of about $130 \mathrm{~nm}$. So far, carbon nanotube-based magnetic junctions are one of the most interesting one-dimensional magnetic junctions, where CNT is sandwiched between magnetic metals [11]. $(6,0)$ zigzag nanotubes have semiconducting properties. There are many works that have been done using $(6,0)$ carbon nanotubes as a barrier in magnetic junction $[11,12]$. In the past, our group has even used $(8,0)$ SiCNTs for the same reasons, as well as 
their popularity [11,12]. It is therefore of interest to use $(6,0) \mathrm{CNT}$ and understand how twisting or stretching of $\mathrm{CNT}$ affect its spin transport properties. The quantum transport properties of pristine $\mathrm{CNT}$ are reported in [13]. It is understood that during the fabrication process some defects may occur in CNTs. Defects such as twisting of CNT, stretching of CNT, and bending of CNT can inevitably occur. CNTs can also be radially expanded and axially elongated during transfer to the substrate. Due to twisting and stretching defects, CNTs can undergo change in the transmission spectrum and band structure. It is therefore of interest to understand how twisting and stretching in CNTs affect its spin transport properties.

In [14], spin filtering in oligopeptide monolayers was investigated, and the authors reported that electron conduction through oligopeptides is spin-dependent. However, in this study, we demonstrate spin-dependent quantum transport properties in twisted carbon nanotube and stretched carbon nanotube. We present a comparative analysis of spin transport in twisted CNT, stretched CNT, and pristine CNT. The current-voltage characteristics, transmission spectrum, magneto resistance, and spin efficiency of twisted and stretched CNTs are calculated using density functional theory (DFT) and non-equilibrium green's function (NEGF). The results are compared with pristine CNT to understand the effect of twisting and stretching on magneto resistance and spin filtration in CNTs.

\section{Simulation Method and Setup}

Figures 1 and 2 show the two-probe geometry of the magnetic junction made up of twisted carbon nanotube and stretched carbon nanotube sandwiched between two $\mathrm{CrO}_{2} \mathrm{HMF}$ electrodes. Figure 3 shows comparison among all the three structures.

The above two-probe geometry is simulated using Atomistix software [15-18] to obtain current-voltage characteristics (see Figures 4 and 5) and transmission spectrum (Figures 6 and 7) in both configurations (PC and APC). The electronic transport properties are calculated using self-consistent calculations. The self-consistent calculations are performed on the basis of DFT and NEGF formulation. For exchange-correlation, spin-polarized generalized gradient approximation (SGGA) is used, because the current is due to spin-polarized electron transport [13]; exact exchange potential of atoms is used to analyze generalized gradient approximations (GGAs) [19]. In general, spin-polarized GGA is more accurate than local-spin density approximation (LSDA) in atomic structure and energetic, but the difference between the former and the latter in terms of the electronic properties of solid-state systems is relatively small [20]. The experimental data is currently unavailable for this structure. Pristine, twisted, and stretched CNTs are also shown in Figure 3.

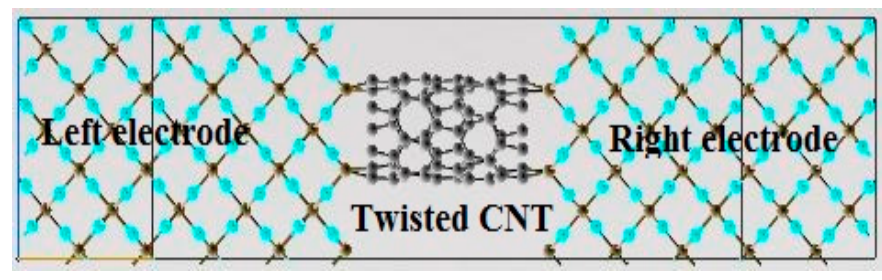

Figure 1. Two-probe geometry in which twisted $\mathrm{CNT}$ is sandwiched between $\mathrm{CrO}_{2}$ electrodes.

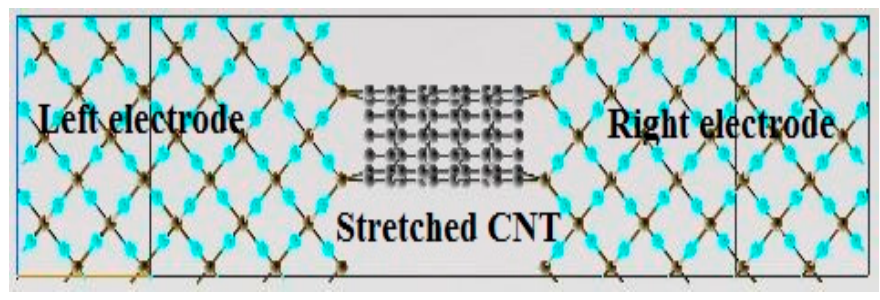

Figure 2. Two-probe geometry in which stretched $\mathrm{CNT}$ is sandwiched between $\mathrm{CrO}_{2}$ electrodes. 


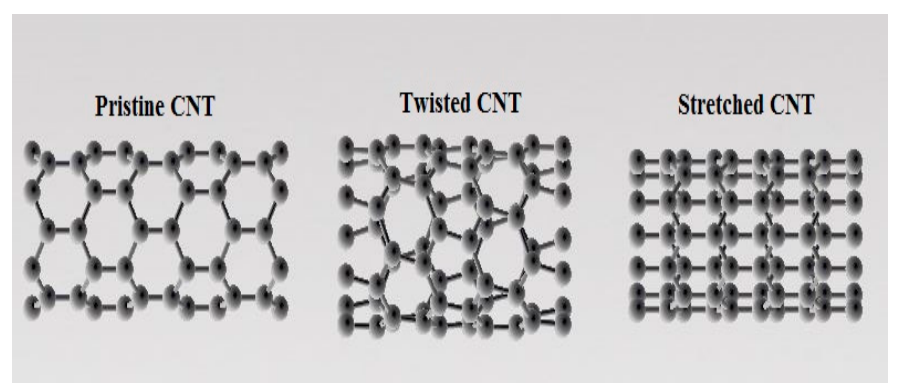

Figure 3. Pristine, twisted, and stretched (in x-y) CNTs.

A zigzag $(6,0)$ twisted CNT and zigzag $(6,0)$ stretched CNT with a bond length of $1.421 \AA$ between carbon atoms and length of $15.629 \AA$ is considered in the simulations, as in [13]. The bond length between carbon and chromium is kept at $2.09 \AA$ [13]. The distance between the electrodes is $19.809 \AA$ The effect may arise from the electric field as well. The authors in [21] have mentioned that the "electric field assisted switching in magnetic tunnel junction". The I-V characteristics and transmission spectrums are acquired by simulating this geometry. The parameters used in the simulation are in accordance with [22-24]. The basis set used is double zeta polarized for carbon nanotube and single zeta polarized for both $\mathrm{CrO}_{2}$ electrodes. The mesh cutoff and electron temperature are assumed to be $150 \mathrm{Ry}$ and $1800 \mathrm{~K}$, respectively. The $\mathrm{k}$ point sampling in $\mathrm{x}, \mathrm{y}$, and $\mathrm{z}$ directions are assumed to be $3,3,100$, respectively, as in [13]. In the simulations, magnetization of the electrodes can be simply changed to either up or down by assuming 1 or -1 spin polarization values. We have done simulation-based studies by assuming parallel magnetization and antiparallel magnetization of the electrodes. The relative spin in a parallel configuration of both electrodes and carbon atoms of twisted or stretched CNT are assumed to be 1, 0,1 . Whereas, in an antiparallel configuration, the right electrode atom spin is assumed to be -1 , so the spin sequence will be $1,0,-1$. The direction of spin polarization — parallel or antiparallel—is in relation to the electron's velocity.

The same is true for the effect of the magnet, how the magnetic field is applied, and what the magnitude of the field is, all of which are demonstrated in the experimental studies reported in [24]. How the resistance depend on field strength is little tricky. When the magnetization of both the electrodes is in same direction (similarly polarized electrodes), potential difference applied across electrodes creates a transverse electric field in the channel (CNT). The higher the potential difference is, the more the electric field in the channel is, and the less the resistance will be. However, in antiparallel polarization alignment of the electrodes - that is, electrode magnetization in opposite direction-the varying electric field will have a negligible change in resistance. One more important observation from the experiments is that high electric fields, when applied to individual electrodes, can reverse their magnetization polarization. The resistance can be low or high, depending upon the relative orientation of ferromagnet magnetization, which can be parallel (P) or antiparallel (AP) [2].

The transmission spectrum indicates the probability of an electron moving from the left electrode to the right electrode under the influence of applied voltage. The current can be evaluated by using the relation [1,15-17]:

$$
\mathrm{I} \uparrow(\downarrow)=e / h \int_{-\infty}^{+\infty} T \uparrow(\downarrow)\left(\mathrm{E}, \mathrm{V}_{\mathrm{B}}\right)\left[\mathrm{F}\left(\mathrm{E}-\mu_{\mathrm{L}}\right)-\mathrm{F}\left(\mathrm{E}-\mu_{\mathrm{R}}\right)\right] \mathrm{dE}
$$

where $\mathrm{T} \uparrow(\downarrow)\left(\mathrm{E}, \mathrm{V}_{\mathrm{B}}\right)$ represents the Transmission coefficient, E as Energy, $\mathrm{F}$ as Fermi-Dirac distribution function, $\mu_{\mathrm{R}}$ and $\mu_{\mathrm{L}}$ are the chemical potential for right and left electrodes. 


\section{Results and Discussion}

For PC and APC configurations, the current-voltage characteristics are shown in Figures 4 and 5. In Figure 4, for PC, I $\downarrow$ is always less than I $\uparrow$ in all the three structures (pristine, twisted, and stretched). The spin-up current increases with an increase in bias, with spin-down current being negligible in all three structures, as shown in Figure 4. The total equilibrium conductance of pristine, twisted and stretched CNTs at zero bias are found to be $0.48 \mathrm{G}_{0}, 0.59 \mathrm{G}_{0}$ and $0.58 \mathrm{G}_{0}$, respectively, where $\mathrm{G}_{0}$ represents conductance quanta, $\mathrm{G}_{0}=2 \mathrm{e}^{2} / \mathrm{h}[1]$.

In Figure 5, both $\mathrm{I} \uparrow$ and $\mathrm{I} \downarrow$ in APC are almost zero for the applied voltage range of 0 to $0.8 \mathrm{~V}$ for all three structures, but spin-up current increases slowly at applied voltages of above $0.8 \mathrm{~V}$ while spin-down current stays around zero. The total equilibrium conductance of pristine, twisted and stretched CNTs is found to be $2.18 \mathrm{G}_{0}, 2.64 \mathrm{G}_{0}$ and $7.0 \mathrm{G}_{0}$, respectively.

The $M R$ is calculated by utilizing the usual definition, $\mathrm{MR}=\left(\mathrm{I}_{\mathrm{PC}}-\mathrm{I}_{\mathrm{APC}}\right) / \mathrm{I}_{\mathrm{PC}}$ [25], where $\mathrm{I}_{\mathrm{PC}}$ and $\mathrm{I}_{\mathrm{APC}}$ are the total currents in parallel configuration and antiparallel configuration. From the current-voltage curve, it can be seen that at zero volts, total current in all three structures is zero, and MR is calculated by utilizing equilibrium conductance. The MR obtained at zero bias is $\sim 100 \%$. At low bias, $\mathrm{I}_{\mathrm{APC}}$ is negligible for all three structures; however, at high bias voltages, $\mathrm{I}_{\mathrm{APC}}$ marginally increases in the case of pristine and twisted CNTs, and is greater in pristine CNT (see Figure 5). For stretched $\mathrm{CNT}$, $\mathrm{I}_{\mathrm{APC}}$ remains negligible even at high bias voltages. This results in higher MR in stretched CNT than in twisted and pristine CNTs (see Figure 8). The physical phenomenon behind the higher MR in stretched and twisted CNTs can be directly associated with the number of electronic states available near Fermi level.

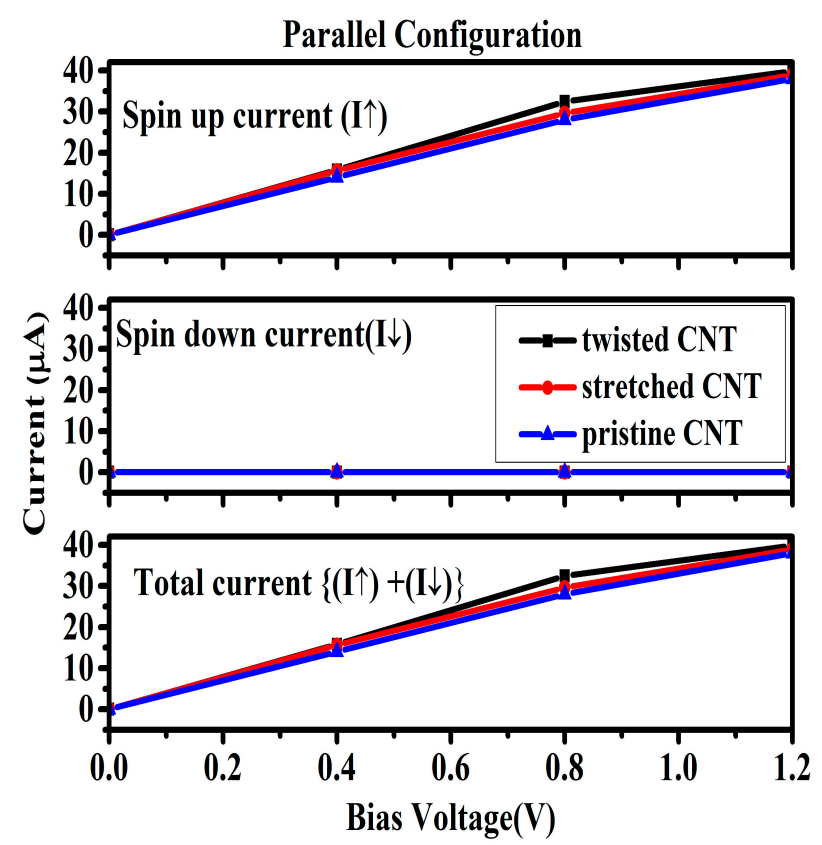

Figure 4. Comparison of current-voltage curves in PC for all three structures. The total spin current of twisted CNT is greater than that of stretched CNT, and the total spin current of stretched CNT is greater than that of pristine CNT. 


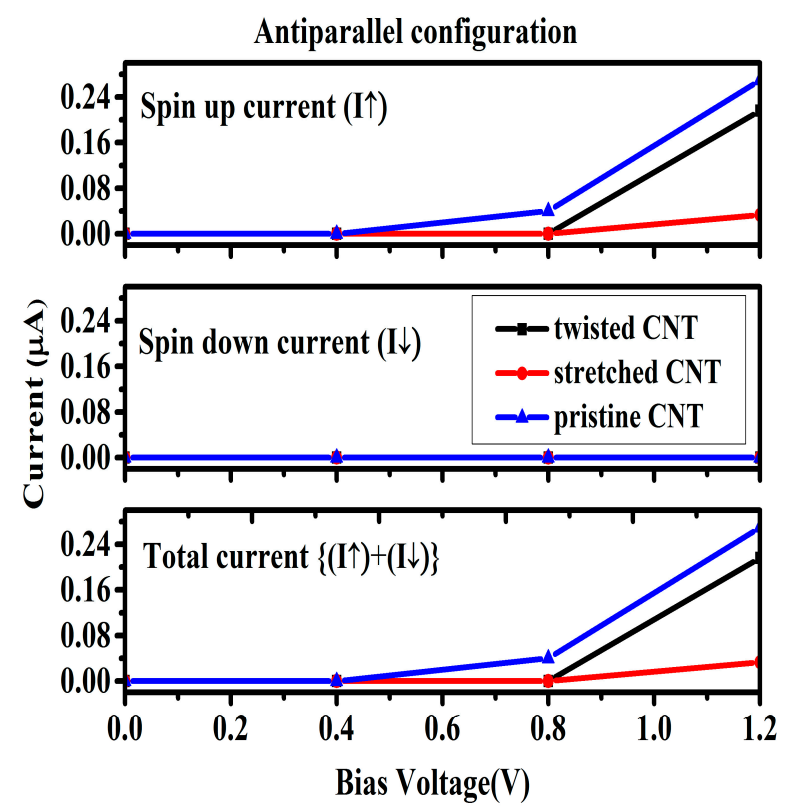

Figure 5. Comparison of I-V curves in APC for all three structures. The total spin current for all three structures is zero for low biases, but for higher biases, spin-down current remains zero, and spin-up current of pristine CNT is greater than that of twisted CNT and the spin-up current of stretched CNT is greater than that of pristine CNT.

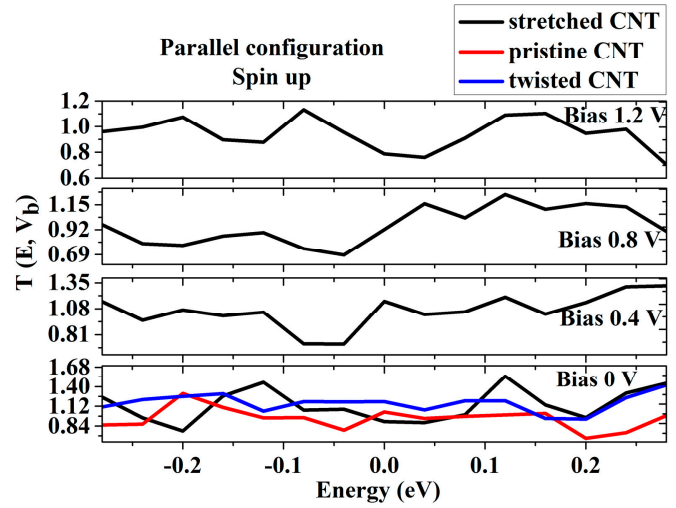

(a)

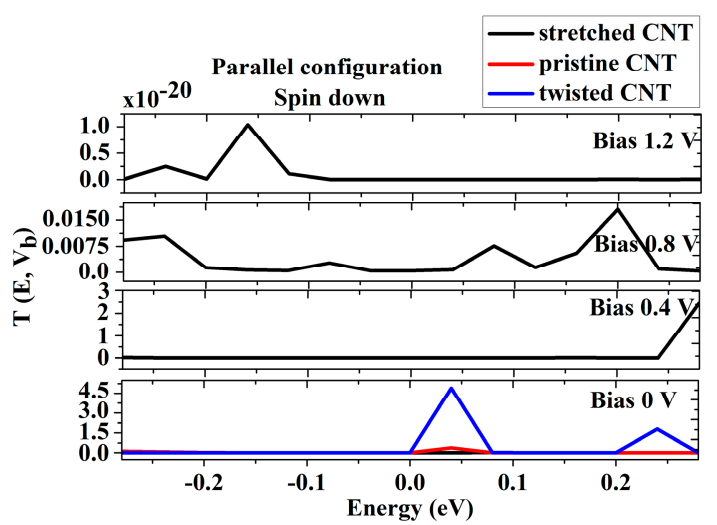

(b)

Figure 6. Transmission spectrum $\mathrm{T}\left(\mathrm{E}, \mathrm{V}_{\mathrm{b}}\right)$ with respect to energy ranging from $-0.3 \mathrm{eV}$ to $+0.3 \mathrm{eV}$ for different applied voltages for PC. At zero volts, the comparative transmission probability for all three structures, shown separately for spin-up (a) and spin-down (b).

There are almost negligible states in all the three structures, in which the current is very small. However, the lowest number of states for twisted structure (see Figure 7a) results in high resistance (see Figure 8) and low $\mathrm{I}_{\mathrm{APC}}$ (see Figure 5). 


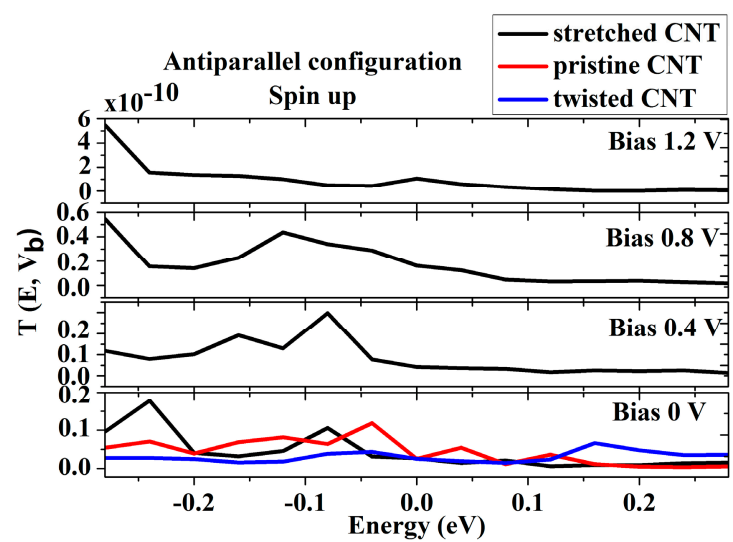

(a)

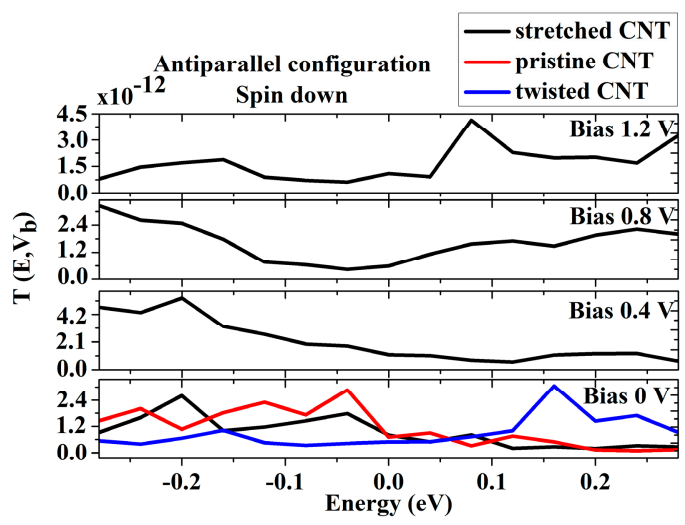

(b)

Figure 7. Transmission spectrum $\mathrm{T}\left(\mathrm{E}, \mathrm{V}_{\mathrm{b}}\right)$ with respect to energy ranging from $-0.3 \mathrm{eV}$ to $+0.3 \mathrm{eV}$ for different applied voltages for APC. At zero volts, the comparative transmission probability for all three structures, shown separately for spin-up (a) and spin-down (b).

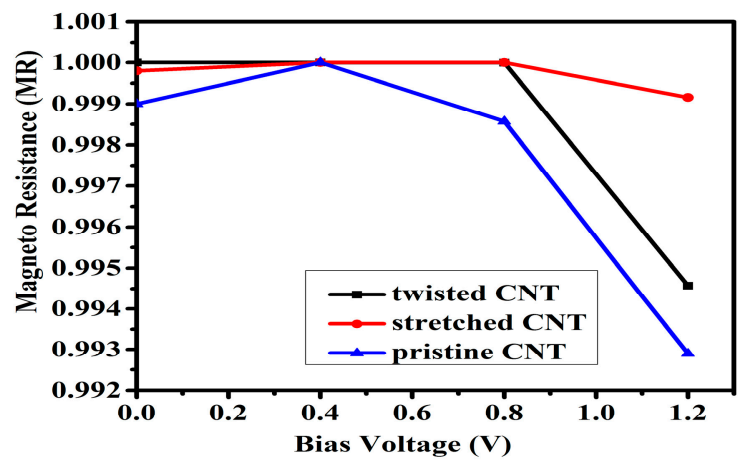

Figure 8. Comparative plots for magneto resistance with respect to applied voltages. For high bias voltage, MR of stretched CNT is higher than that of twisted CNT, and MR of twisted CNT is higher than that of pristine CNT.

Spin injection factor $(\eta)$ is calculated by utilizing the following definition: $\eta=(\mathrm{I} \uparrow-\mathrm{I} \downarrow) /(\mathrm{I} \uparrow+\mathrm{I} \downarrow)[2]$, and is plotted in Figure 9. At zero volts, total current in all three structures is zero, and spin injection factor is calculated by utilizing equilibrium conductance. Parallel configuration $\mathrm{I} \uparrow$ is nearly equal, and $\mathrm{I} \downarrow$ is negligible in all three cases (see Figure 4), resulting in only carriers with up-spin being allowed to propagate through the CNT, and blocking down-spin carriers, resulting in high spin filtration ( $\eta$ ) in the case of PC at all bias points.

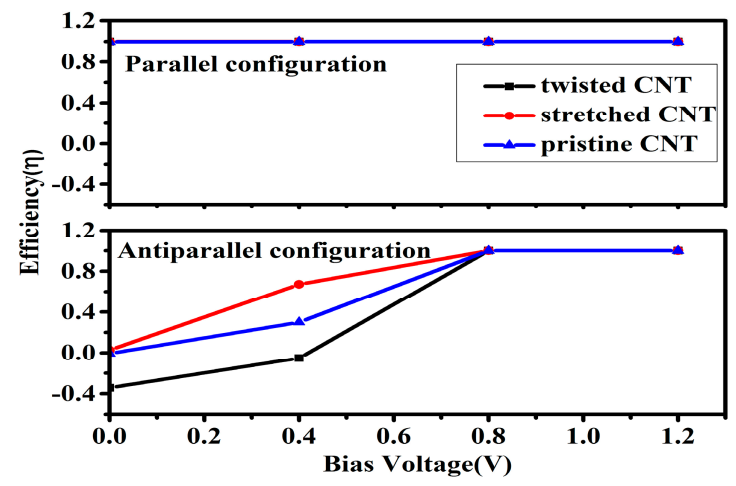

Figure 9. Spin injection factor with respect to applied voltages for all three structures in parallel configuration and antiparallel configuration. 
In APC, stretching increases the spin filtration at all bias points in comparison to pristine and twisted structures, and is $\sim 100 \%$ at high bias voltages. However, twisting results in lower spin filtration than in pristine and stretched CNTs at low bias voltages, and also negative spin filtration (see Figure 9) due to $\mathrm{I} \downarrow$ being greater than $\mathrm{I} \uparrow$.

\section{Conclusions}

The spin-dependent quantum transport properties in twisted CNT and stretched CNT are investigated, and it is found that the magneto resistance (MR) of stretched CNT is higher than that of twisted CNT, and the MR of twisted CNT is higher than that of pristine CNT, and that higher spin filtration is obtained in stretched CNT in comparison to pristine CNT. High MR and high spin filtration show the importance of twisted and stretched carbon nanotube for use in spin valves and other applications.

Author Contributions: Anil Kumar Singh performed the experiment, analyzed the data and wrote the paper under the guidance of Sudhanshu Choudhary.

Conflicts of Interest: The authors declare no conflict of interest.

\section{References}

1. Yao, K.L.; Min, Y.; Liu, Z.L.; Cheng, H.G.; Zhu, S.C.; Gao, G.Y. First-principles study of transport of V doped boron nitride nanotube. Phys. Lett. A 2008, 372, 5609-5613. [CrossRef]

2. Titus, E.; Krishna, R.; Grácio, J.; Singh, M.; Ferreira, A.L.; Dias, R.G. Carbon nanotube based magnetic tunnel junctions (MTJs) for spintronics application, Electronic Properties of Carbon Nanotube. InTech 2011. [CrossRef]

3. Bratkovsky, A.M. Tunneling of electrons in conventional and half-metallic systems towards very large magnetoresistance. Phys. Rev. B 1997, 56, 2344. [CrossRef]

4. Anguelouch, A.; Gupta, A.; Xiao, G.; Abraham, D.W.; Ji, Y.; Ingvarsson, S.; Chien, C.L. Near-complete spin polarization in atomically-smooth chromium-dioxide epitaxial films prepared using a CVD liquid precursor. Phys. Rev. B 2001, 64, 180408. [CrossRef]

5. Miao, G.X.; LeClair, P.; Gupta, A.; Xiao, G.; Varela, M.; Pennycook, S. Magnetic tunnel junctions based on $\mathrm{CrO}_{2} / \mathrm{SnO}_{2}$ epitaxial bilayers. Appl. Phys. Lett. 2006, 89, 022511. [CrossRef]

6. Fu, Q.F.; Hao, D.P.; Yan, X.M.; He, D.W.; Chen, Z.S.; Wang, L.G.; Terence, K.S. Electronics properties of Single-walled Twisted carbon nanotubes. In Proceedings of the 2011 IEEE Fourth International Conference on Information and Computing (ICIC), Phuket Island, Thailand, 25-27 April 2011.

7. Mintmire, J.W.; Dunlap, B.I.; White, C.T. Are fullerene tubes metallic. Phys. Rev. Lett. 1992, 68, 631-634. [CrossRef] [PubMed]

8. Hamada, N.; Sawada, S.; Oshiyama, A. New one-dimensional conductors: Graphitic microtubules. Phys. Rev. Lett. 1992, 68, 1579-1581. [CrossRef] [PubMed]

9. Saito, R.; Fujita, M.; Dresselhaus, G.; Dresselhaus, M.S. Electronic structure of chiral graphene tubules. Appl. Phys. Lett. 1992, 60, 2204-2206. [CrossRef]

10. Saito, R.; Fujita, M.; Dresselhaus, G.; Dresselhaus, M.S. Electronic structure of graphene tubules based on $\mathrm{C}_{60}$. Phys. Rev. B 1992, 46, 1804-1811. [CrossRef]

11. Wang, B.; Zhu, Y.; Ren, W.; Wang, J.; Guo, H. Spin-dependent transport in Fe-doped carbon nanotubes. Phys. Rev. B 2007, 75, 235415. [CrossRef]

12. Zhao, B.; Monch, I.; Vinzelberg, H.; Muhl, T.; Schneider, C.M. Spin-coherent transport in ferromagnetically contacted carbon nanotubes. Appl. Phys. Lett. 2002, 80, 3144. [CrossRef]

13. Choudhary, S.; Varshney, M. First-Principles Study of Spin Transport in $\mathrm{CrO}_{2}-\mathrm{CNT}-\mathrm{CrO}_{2}$ Magnetic Tunnel Junction. J. Superconduct. Novel Magn. 2015, 28, 3141-3145. [CrossRef]

14. Atomistix, QuantumWise A/S. Available online: www.quantumwise.com (accessed on 1 April 2011).

15. Brandbyge, M.; Mozos, J.L.; Ordejón, P.; Taylor, J.; Stokbro, K. Density-functional method for nonequilibriun electron transport. Phys. Rev. B 2002, 65, 165401. [CrossRef]

16. Taylor, J.; Guo, H.; Wang, J. Ab initio modeling of quantum transport properties of molecular electronic devices. Phys. Rev. B 2001, 63, 245407. [CrossRef] 
17. José, M.; Emilio Artacho, S.; Gale, J.D.; García, A.; Junquera, J.; Ordejón, P.; Sánchez-Portal, D. The SIESTA method for ab initio order-N materials simulation. J. Phys. Condens. Matter 2002, 14, 2745-2779.

18. Engel, E.; Vosko, S.H. Exact exchange-only potentials and the virial relation as microscopic criteria for generalized gradient approximations. Phys. Rev. B 1993, 47, 13164. [CrossRef]

19. Lee, I.-H.; Martin, R.M. Applications of the generalized-gradient approximation to atoms, clusters, and solids. Phys. Rev. B 1997, 56, 7197. [CrossRef]

20. Wang, W.G.; Li, M.; Hageman, S.; Chien, C.L. Electric-field-assisted switching in magnetic tunnel junctions. Nat. Mater. 2012, 11, 64. [CrossRef] [PubMed]

21. Nowak, J.; Rauluszkiewicz, J. Spin dependent electron tunneling between ferromagnetic films. J. Magn. Magn. Mater. 1992, 109, 79-90. [CrossRef]

22. Choudhary, S.; Qureshi, S. Theoretical study on transport properties of a BN co-doped SiC nanotube. Phys. Lett. A 2011, 375, 3382-3385. [CrossRef]

23. Poklonski, N.A.; Ratkevich, S.V.; Vyrko, S.A.; Kislyakov, E.F.; Bubel, O.N.; Popov, A.M.; Lozovik, Y.E.; Hieu, N.N.; Viet, N.A. Structural phase transition and bandgap of uniaxiallydeformed $(6,0)$ carbon nanotube. Chem. Phys. Lett. 2012, 545, 71-77. [CrossRef]

24. Chakraverty, M.; Kittur, H.M.; Arun Kumar, P. First principle simulations of various magnetic tunnel junctions for applications in magnetoresistive random access memories. IEEE Trans. Nanotechnol. 2013, 6, 12.

25. Kiran, V.; Cohen, S.R.; Naaman, R. Structure dependent spin selectivity in electron transport through oligopeptides. J. Chem. Phys. 2017, 146, 092302. [CrossRef]

(C) 2017 by the authors. Licensee MDPI, Basel, Switzerland. This article is an open access article distributed under the terms and conditions of the Creative Commons Attribution (CC BY) license (http:/ / creativecommons.org/licenses/by/4.0/). 